Article

\title{
Propagation of Optical Coherence Vortex Lattices in Turbulent Atmosphere
}

\author{
Yan Huang ${ }^{1}$, Yangsheng Yuan ${ }^{2, *}$, Xianlong Liu ${ }^{2}{ }^{\circledR}$, Jun Zeng ${ }^{1}$, Fei Wang ${ }^{1}$, Jiayi Yu ${ }^{1}$, Lin Liu ${ }^{1}$ \\ and Yangjian Cai ${ }^{1,2, *}$ \\ 1 School of Physical Science and Technology, Soochow University, Suzhou 215006, China; \\ 20164208026@stu.suda.edu.cn (Y.H.); jzeng@stu.suda.edu.cn (J.Z.); fwang@suda.edu.cn (F.W.); \\ jyyu@stu.suda.edu.cn (J.Y.); liulin@suda.edu.cn (L.L.) \\ 2 Center of Light Manipulations and Applications \& Shandong Provincial Key Laboratory of Optics and \\ Photonic Device, School of Physics and Electronics, Shandong Normal University, Jinan 250014, China; \\ xianlongliu@sdnu.edu.cn \\ * Correspondence: yysheng@sdnu.edu.cn (Y.Y.); yangjiancai@suda.edu.cn (Y.C.); \\ Tel.: +86-531-89611187 (Y.Y. \& Y.C.)
}

Received: 30 October 2018; Accepted: 27 November 2018; Published: 3 December 2018

\begin{abstract}
Propagation properties in the turbulence atmosphere of the optical coherence vortex lattices (OCVLs) are explored by the recently developed convolution approach. The evolution of spectral density distribution, the normalized $M^{2}$-factor, and the beam wander of the OCVLs propagating through the atmospheric turbulence with Tatarskii spectrum are illustrated numerically. Our results show that the OCVLs display interesting propagation properties, e.g., the initial Gaussian beam distribution will evolve into hollow array distribution on propagation and finally becomes a Gaussian beam spot again in the far field in turbulent atmosphere. Furthermore, the OCVLs with large topological charge, large beam array order, large relative distance, and small coherence length are less affected by the negative effects of turbulence. Our results are expected to be used in the complex system optical communications.
\end{abstract}

Keywords: optical coherence vortex lattices; atmospheric turbulence; evolution properties; free-space optical communications

\section{Introduction}

Optical lattices can be viewed as a typical kind of beam arrays, which display sufficiently small beam spots. Recently, a novel class of optical lattices, which are named optical coherence lattices (OCLs), were introduced by Ma and Ponomarenko [1]. The OCLs were described with the help of the complex Gaussian representation and the time-domain OCLs were generated through superposition of Gaussian pulses [2]. The property of periodic reciprocity of the OCLs on propagation through the free-space was found [3]. Periodic reciprocity means the periodic degree of coherence at the source plane transfers the periodicity to the transverse intensity distribution after a long propagation distance. Recently, both scalar and vector OCLs were generated in experiment [4,5]. Furthermore, other kinds of optical lattices were also proposed [6-8]. The optical lattices were found to be useful in various applications, e.g., image transmission and encryption [4], trapping and cooling atoms [9], photonic crystals engineering [10], lattice light-sheet microscopy [11], and ultracold trapping of quantum gas [12].

On the other hand, partially coherent beams have been studied extensively over the last few decades due to their novel physical properties and widely uses in various fields, such as free-space optical communications, optical imaging, particle trapping, particle scattering, and remote 
detection. The statistical properties, e.g., degree of coherence [13], beam spreading [14,15], propagation factor [16-18], Rayleigh range [19], and scintillation index [20-22] of different kinds of partially coherent beams in free space and in various turbulent atmosphere have been studied in detail. The propagation properties of the OCLs (i.e., a typical kind of partially coherent beams with periodical degree of coherence) in atmospheric turbulence were explored [23], and the results showed that the OCLs have an advantage of mitigating scintillation index in atmospheric turbulence on propagation.

A vortex beam with twisted wavefront carries an orbital angular momentum and has various applications ranging from optical tweezers to optical communications [24-28]. The topological charge (which defines how the wavefront of the vortex beam twists) on propagation in atmospheric turbulence is robust [29]. In 1998, Gori et al. introduced a class of partially coherent beams with helical wavefront, which were named partially coherent vortex beams (PCVB) [30]. Subsequently, efforts have been made to the PCVBs [31-44]. A review on propagation, generation, and measurement of PCVBs was given [41], and self-healing properties of a PCVB was found [42]. More recently, PCVB with a fractional topological charge [43] and partially coherent vortex beam with periodical degree of coherence (i.e., optical coherence vortex lattices) [44] were introduced, respectively. In this paper, we investigate the statistical properties (i.e., spectral density distribution, normalized $\mathrm{M}^{2}$-factor, and beam wander) of optical coherence vortex lattices (OCVLs) in turbulent atmosphere by using the recently developed convolution approach. We find that the initial Gaussian beam distribution of the OCVLs evolves into hollow array distribution on propagation and finally becames a Gaussian beam spot again in the far field in turbulent atmosphere. The OCVLs with prescribed beam parameters are less affected by the turbulence, which will be useful for free-space optical communications.

\section{Formulation of the Propagation of OCLVs in Turbulent Atmosphere}

The second-order properties of a partially coherent beam in frequency domain can be studied by the cross-spectral density (CSD) function. To construct a bona fide field, the CSD function at source can be expanded as follows [45]:

$$
W\left(\mathbf{r}_{1}, \mathbf{r}_{2} ; 0\right)=\int p(\mathbf{v}) H^{*}\left(\mathbf{r}_{1}, \mathbf{v}\right) H\left(\mathbf{r}_{2}, \mathbf{v}\right) d^{2} \mathbf{v},
$$

where $\mathbf{r}_{1}=\left(x_{1}, y_{1}\right)$ and $\mathbf{r}_{2}=\left(x_{2}, y_{2}\right)$ are the two arbitrary transverse position vectors at source plane, $p(\mathbf{v})$ is a scalar nonnegative function, $H(\mathbf{r}, \mathbf{v})$ denotes an arbitrary suitable kernel function.

To generate OCVLs, we define $p(\mathbf{v})$ and $H(\mathbf{r}, \mathbf{v})$ as follows:

$$
\begin{gathered}
p(\mathbf{v})=\frac{1}{M_{m}} \sum_{m-(M-1) / 2}^{(M-1) / 2} \exp \left[-\frac{\left|\mathbf{v}-\mathbf{v}_{0 m}\right|^{2}}{2 w_{0}^{2}}\right], \\
H(\mathbf{r}, \mathbf{v})=-\frac{i}{\lambda f} \tau(\mathbf{r}) \exp \left[\frac{i \pi}{\lambda f}\left(\mathbf{v}^{2}-2 \mathbf{r} \cdot \mathbf{v}\right)\right] .
\end{gathered}
$$

where $p(\mathbf{v})$ is superposed by the $M$ space-shifted Gaussian functions, $w_{0}$ and $\mathbf{v}_{0 m}=(m d, m d)$ denote the circular aperture radius and the position vector of the lattices, respectively, and $d$ denotes the distance between adjacent spots. $H(\mathbf{r}, \mathbf{v})$ represents in the experiment the response function of an optical Fourier transform system consisting of a free-space distance of $f$, a thin lens with focal distance $f$, and an optical filter with transmission function $\tau(\mathbf{r})$ [44]. Here we set $\tau(\mathbf{r})$ in the cylindrical coordinates as follows:

$$
\tau(r, \theta)=\left(\frac{\sqrt{2} r}{\omega_{0}}\right)^{l} \exp \left(-\frac{r^{2}}{\omega_{0}^{2}}\right) \exp (-i l \theta),
$$

where $l$ and $\omega_{0}$ are the topological charge and beam width, respectively, $(r, \theta)$ are cylindrical polar coordinates. 
By substituting Equations (2)-(4) into Equation (1), the CSD of the OCVLs at source plane is obtained as follows:

$$
\begin{aligned}
W_{0}\left(r_{1}, \theta_{1}, r_{2}, \theta_{2}\right) & =\frac{2 w_{0}^{2} \pi}{M \lambda^{2} f^{2}} \sum_{m=-(M-1) / 2}^{(M-1) / 2}\left(\frac{2 r_{1} r_{2}}{\omega_{0}^{2}}\right)^{l} \exp \left(-\frac{r_{1}^{2}+r_{2}^{2}}{\omega_{0}^{2}}\right) \exp \left[i l\left(\theta_{2}-\theta_{1}\right)\right] \\
& \times \exp \left[-\frac{r_{1}^{2}+r_{2}^{2}-2 r_{1} r_{2} \cos \left(\theta_{2}-\theta_{1}\right)}{2 \delta_{0}^{2}}\right] \\
& \times \exp \left\{-b\left[\left(r_{2} \cos \theta_{2}-r_{1} \cos \theta_{1}+r_{2} \sin \theta_{2}-r_{1} \sin \theta_{1}\right)\right]\right\},
\end{aligned}
$$

where $\delta_{0}=f /\left(w_{0} k\right)$ is the beam initial coherence width. For the convenience of calculation, we set $a=1 / 2 \delta_{0}^{2}=2 w_{0}^{2} \pi^{2} /\left(\lambda^{2} f^{2}\right), b=2 i \pi m d /(\lambda f)$.

The spectral density of a propagating partially coherent beam in turbulent atmosphere can be obtained by using the convolution approach as follows [46]:

$$
S(\boldsymbol{\rho}, z)=S_{f}(-\boldsymbol{\rho} / z) \otimes p_{1}(-\boldsymbol{\rho} / z) \otimes p_{2}(-\boldsymbol{\rho} / z)
$$

where $\rho$ is the position vector in the receiver plane with the propagation distance being $z . S_{f}(-\rho / z)$ is the spectral density of the incoherent portion on propagation in free space, $p_{1}(-\rho / z)$ is the Fourier transform of the degree of coherence of the beam at source plane, $p_{2}(-\rho / z)$ is the Fourier transform of second-order random phase correlation function, and $\otimes$ is the symbol of convolution.

According to the Huygens-Fresnel integral, $S_{f}(-\rho / z)$ is obtained by:

$$
S_{f}(-\rho / z)=|\widetilde{A}(\boldsymbol{\rho} / z)|^{2} / \lambda^{2} z^{2}
$$

where $A(\mathbf{r})=\tau(\mathbf{r}) \exp \left(i k \mathbf{r}^{2} / 2 z\right)$ and the hat tilde denotes the two-dimensional Fourier transform, and [47]:

$$
p_{2}\left(\mathbf{v}^{\prime}\right)=\frac{3}{2 \pi^{3} T z} \exp \left(-\frac{3 \mathbf{v}^{\prime 2}}{2 \pi^{2} T z}\right)
$$

with:

$$
T=\int_{-\infty}^{\infty} \kappa^{3} \Phi_{n}(\kappa) d \kappa
$$

Here $\Phi_{n}(\kappa)$ is the power spectrum density of the atmospheric turbulence refractive index fluctuations, $\kappa$ denotes the frequency in the space. Here we adopted the Tatarskii spectrum for the power spectrum density $\Phi_{n}(\kappa)$, which is written as [48]:

$$
\Phi_{n}(\kappa)=0.033 C_{n}^{2} \kappa^{-11 / 3} \exp \left(-\frac{\kappa^{2}}{\kappa_{m}^{2}}\right) .
$$

Here $C_{n}^{2}$ denotes the structure parameter of the atmospheric turbulence and $\kappa_{m}=5.92 / l_{0}$ with $l_{0}$ being the turbulence inner scale. In this paper, we set $l_{0}=0.01 \mathrm{~m}$. Taking Equation (10) into Equation (9), we obtain:

$$
T=0.771 C_{n}^{2}
$$

Taking Equations (2), (4), (7), and (8) into Equation (6), and using the fast Fourier transform (FFT) algorithm, one can numerically analyze the spectral density of the OCVLs propagating in turbulent atmosphere by using MATLAB (The MathWorks, Natick, MA, USA).

For further investigating the propagation properties of the OCVLs, we also study the $M^{2}$-factor and beam wander [49-51]. The beam's second-order moments in turbulent atmosphere in the receiver plane are obtained as follows [51]:

$$
\left\langle r^{2}\right\rangle=\left\langle r^{2}\right\rangle_{0}+2\langle\mathbf{r} \cdot \theta\rangle_{0} z+\left\langle\theta^{2}\right\rangle_{0} z^{2}+\frac{4}{3} \pi^{2} T z^{3}
$$




$$
\begin{gathered}
\left\langle\theta^{2}\right\rangle=\left\langle\theta^{2}\right\rangle_{0}+4 \pi^{2} T z, \\
\langle\mathbf{r} \cdot \boldsymbol{\theta}\rangle=\langle\mathbf{r} \cdot \boldsymbol{\theta}\rangle_{0}+\left\langle\theta^{2}\right\rangle_{0} z+2 \pi^{2} T z^{2},
\end{gathered}
$$

By the partial derivative method, the second-order moments of the partially coherent beams at source plane can be obtained by [52]:

$$
\begin{aligned}
& \left\langle r^{2}\right\rangle_{0}=\frac{1}{I} \int_{0}^{2 \pi} \int_{0}^{\infty} r^{3} W_{0}(r, \theta, r, \theta) d r d \theta, \\
& \left\langle\theta^{2}\right\rangle_{0}=\frac{1}{k^{2} I} \int_{0}^{2 \pi} \int_{0}^{\infty}\left\{\frac{\partial^{2} W_{0}\left(r_{1}, \theta_{1}, r_{2}, \theta_{2}\right)}{\partial r_{1} \partial r_{2}}\left|\begin{array}{l}
r_{1}=r_{2}=r \\
\theta_{1}=\theta_{2}=\theta
\end{array}+\frac{1}{r^{2}} \frac{\partial^{2} W_{0}\left(r_{1}, \theta_{1}, r_{2}, \theta_{2}\right)}{\partial \theta_{1} \partial \theta_{2}}\right| \begin{array}{l}
r_{1}=r_{2}=r \\
\theta_{1}=\theta_{2}=\theta
\end{array}\right\} r d r d \theta, \\
& \langle r \theta\rangle_{0}=\frac{1}{i k I} \int_{0}^{2 \pi} \int_{0}^{\infty}\left\{r_{1} \frac{\partial W_{0}\left(r_{1}, \theta_{1}, r_{2}, \theta_{2}\right)}{\partial r_{1}}-r_{2} \frac{\partial W_{0}\left(r_{1}, \theta_{1}, r_{2}, \theta_{2}\right)}{\partial r_{2}}\right\} \mid \begin{array}{l}
r_{1}=r_{2}=r \quad r d r d \theta, \\
\theta_{1}=\theta_{2}=\theta
\end{array}
\end{aligned}
$$

where $k=2 \pi / \lambda$ denotes the wavenumber, and:

$$
I=\int_{0}^{2 \pi} \int_{0}^{\infty} W(r, \theta, r, \theta ; 0) r d r d \theta
$$

is the total energy for the beam.

By substituting Equations (5), (15)-(17) into Equations (12)-(14), we obtained:

$$
\begin{gathered}
\left\langle r^{2}\right\rangle=\frac{\omega_{0}^{2}(l+1)}{2}+\frac{\lambda^{2} z^{2}}{4 \pi^{2}}\left[4 a+\frac{2(l+1)}{\omega_{0}^{2}}-\frac{2}{M} \sum_{m=-(M-1) / 2}^{(M-1) / 2} b^{2}\right]+\frac{4}{3} \pi^{2} T z^{3}, \\
\left\langle\theta^{2}\right\rangle=\frac{\lambda^{2}}{4 \pi^{2}}\left[4 a+\frac{2(l+1)}{\omega_{0}^{2}}-\frac{2}{M} \sum_{m=-(M-1) / 2}^{(M-1) / 2} b^{2}\right]+4 \pi^{2} T z, \\
\langle r \theta\rangle=\frac{\lambda^{2} z}{4 \pi^{2}}\left[4 a+\frac{2(l+1)}{\omega_{0}^{2}}-\frac{2}{M} \sum_{m=-(M-1) / 2}^{(M-1) / 2} b^{2}\right]+2 \pi^{2} T z^{2} .
\end{gathered}
$$

The $M^{2}$-factor is an important parameter to measure the laser quality and is defined as [51-53]:

$$
M^{2}(z)=\frac{2 \pi}{\lambda}\left(\left\langle r^{2}\right\rangle\left\langle\theta^{2}\right\rangle-\langle r \theta\rangle^{2}\right)^{1 / 2}
$$

The $M^{2}$-factor of OCVLs can be obtained by substituting Equations (19)-(21) into Equation (22). Our results agree well with the Ref. [40] when $M=1$ and $l=0$.

According to Ref. [51-53], we obtain the following expressions for the effective beam size:

$$
W_{L T}=\sqrt{\frac{\omega_{0}^{2}(l+1)}{2}+\frac{\lambda^{2} z^{2}}{4 \pi^{2}}\left[4 a+\frac{2(l+1)}{\omega_{0}^{2}}-\frac{2}{M} \sum_{m=-(M-1) / 2}^{(M-1) / 2} b^{2}\right]+\frac{4}{3} \pi^{2} T z^{3}}
$$

and in free space

$$
W_{F S}=\sqrt{\frac{\omega_{0}^{2}(l+1)}{2}+\frac{\lambda^{2} L^{2}}{4 \pi^{2}}\left[4 a+\frac{2(l+1)}{\omega_{0}^{2}}-\frac{2}{M} \sum_{m=-(M-1) / 2}^{(M-1) / 2} b^{2}\right]}
$$


Beam wander is characterized by $\left\langle r_{c}^{2}\right\rangle^{1 / 2}$ of the variance of off-axis displacement of the instantaneous beam center. Beam wander is defined as follows [54]:

$$
\left\langle r_{c}^{2}\right\rangle=\frac{16 \pi^{3}}{\lambda^{2}} W_{F S}^{2} \int_{0}^{L} \int_{0}^{\infty} \kappa \Phi_{n}(\kappa) \exp \left(-\kappa^{2} W_{L T}^{2}\right)\left\{1-\exp \left[\frac{-2 L^{2} \kappa^{2}(1-z / L)}{k^{2} W_{F S}^{2}}\right]\right\} d \kappa d z,
$$

taking Equation (10) into Equation (25), Equation (25) is simplified as:

$$
\left\langle r_{c}^{2}\right\rangle=\frac{0.132}{\lambda} \pi^{3} W_{F S}^{2} \Gamma(-5 / 6) \int_{0}^{L}\left\{\left(2.85 \times 10^{-6}+W_{L T}^{2}\right)^{5 / 6}-\left[\frac{2 L^{2}(1-z / L)^{2}}{k^{2} W_{F S}^{2}}+2.85 \times 10^{-6}+W_{L T}^{2}\right]^{5 / 6}\right\} d z,
$$

the evolution properties of the beam wander in turbulent atmosphere of OCVLs can be calculated numerically by taking Equations (23) and (24) into Equation (26).

\section{Numerical Results}

\subsection{Spectral Density Distribution}

Figure 1 displays the spectral density distribution of the OCVLs in turbulent atmosphere at various propagation distances with $\lambda=532.8 \mathrm{~nm}, M=3, l=2, \omega_{0}=1 \mathrm{~mm}, \delta_{0}=3.4 \mathrm{~mm}, C_{n}^{2}=$ $10^{-13} \mathrm{~m}^{-2 / 3}$, and $d=0.3 \mathrm{~mm}$. From Figure 1a), one finds that the spectral density distribution of the OCVLs at source is a single beam spot with doughnut beam profile. The single beam spot gradually evolves into $3 \times 3$ hollow beam arrays (see Figure 1e) with the increase of the propagation distance $z$, which is similar to its evolution properties in free-space [44]. However, when we further increase the propagation distance, the effect of turbulence on beam properties accumulates gradually. The hollow beam arrays disappear gradually and finally become a Gaussian beam profile in the far field (see Figure 1h).
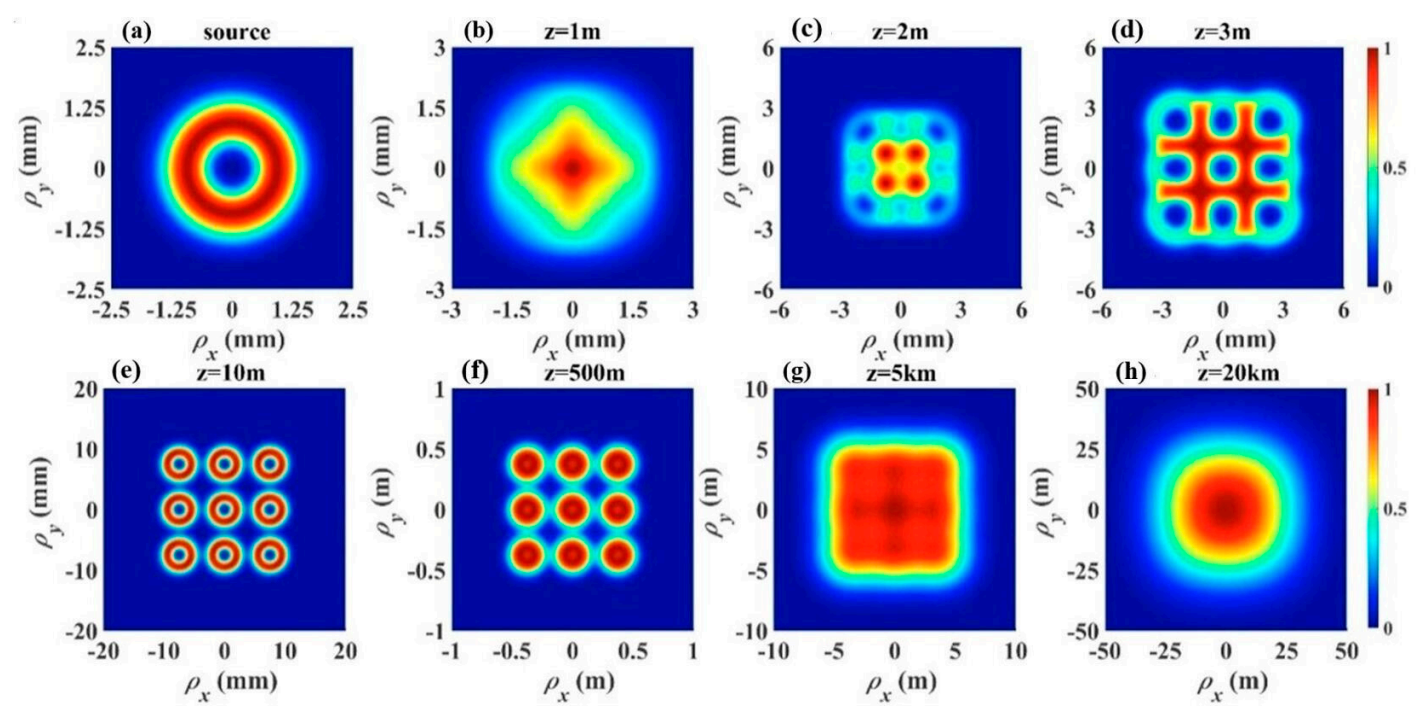

Figure 1. Spectral density distribution at several $z$ of the optical coherence vortex lattices in turbulent atmosphere with $\lambda=532.8 \mathrm{~nm}, M=3, l=2, \omega_{0}=1 \mathrm{~mm}, \delta_{0}=3.4 \mathrm{~mm}, C_{n}^{2}=10^{-13} \mathrm{~m}^{-2 / 3}$, and $d=$ $0.3 \mathrm{~mm}$. (a) $z=0$; (b) $z=1 \mathrm{~m}$; (c) $z=2 \mathrm{~m}$; (d) $z=3 \mathrm{~m}$; (e) $z=10 \mathrm{~m}$; (f) $z=500 \mathrm{~m} ;(\mathbf{g}) z=5 \mathrm{~km}$; (h) $z=20 \mathrm{~km}$.

Figure 2 displays the spectral density distribution of the OCVLs in turbulent atmosphere at $z=$ $5 \mathrm{~km}$ for several values of the relative distance with $\lambda=532.8 \mathrm{~nm}, M=3, l=2, \omega_{0}=1 \mathrm{~mm}, \delta_{0}=$ $3.4 \mathrm{~mm}$, and $C_{n}^{2}=10^{-13} \mathrm{~m}^{-2 / 3}$. From Figure 2, one sees that the beam distribution of the OCVLs evolves into Gaussian distribution rapidly when the relative distance $d$ is small (see Figure 2a). On the contrary, the beam array profile persists for longer distances when the relative distance $d$ increases 
(see Figure 2d). Figure 3 displays the spectral density of the OCVLs in turbulent atmosphere at $z=5 \mathrm{~km}$ for several values of the array index $M$ with $\lambda=532.8 \mathrm{~nm}, l=2, \delta_{0}=0.45 \mathrm{~mm}, \omega_{0}=$ $1 \mathrm{~mm}, C_{n}^{2}=10^{-13} \mathrm{~m}^{-2 / 3}$, and $d=0.3 \mathrm{~mm}$. From Figure 3, the beam spot size of the OCVLs increases as the array order $M$ increases, which indicates the OCVLs spread more rapidly as the array order $M$ increases. Figure 4 displays the spectral density distribution of the OCVLs in turbulent atmosphere at $z=500 \mathrm{~m}$ for several values of $l$ with $\lambda=532.8 \mathrm{~nm}, M=3, \delta_{0}=0.7 \mathrm{~mm}, \omega_{0}=1 \mathrm{~mm}, C_{n}^{2}=$ $10^{-13} \mathrm{~m}^{-2 / 3}$, and $d=1 \mathrm{~mm}$ (see Figure 4a). One finds that the beam arrays keep hollow profiles for longer propagation distances as the topological charge $l$ increases, which means the OCVLs with large topological charges are less affected by turbulence.
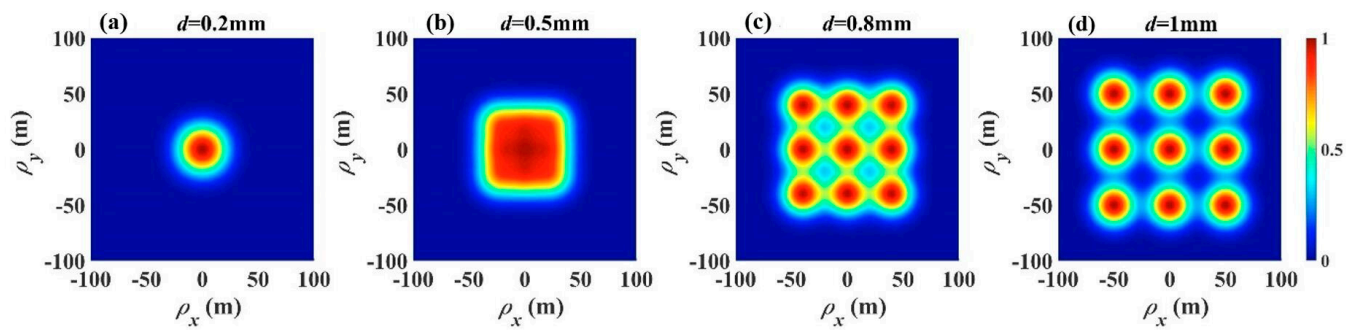

Figure 2. Spectral density distribution of the optical coherence vortex lattices in turbulent atmosphere at $z=5 \mathrm{~km}$ for different $d$ with $\lambda=532.8 \mathrm{~nm}, M=3, l=2, \omega_{0}=1 \mathrm{~mm}, \delta_{0}=3.4 \mathrm{~mm}$, and $C_{n}^{2}=$ $10^{-13} \mathrm{~m}^{-2 / 3}$. (a) $d=0.2 \mathrm{~mm}$; (b) $d=0.5 \mathrm{~mm}$; (c) $d=0.8 \mathrm{~mm}$; (d) $d=1 \mathrm{~mm}$.
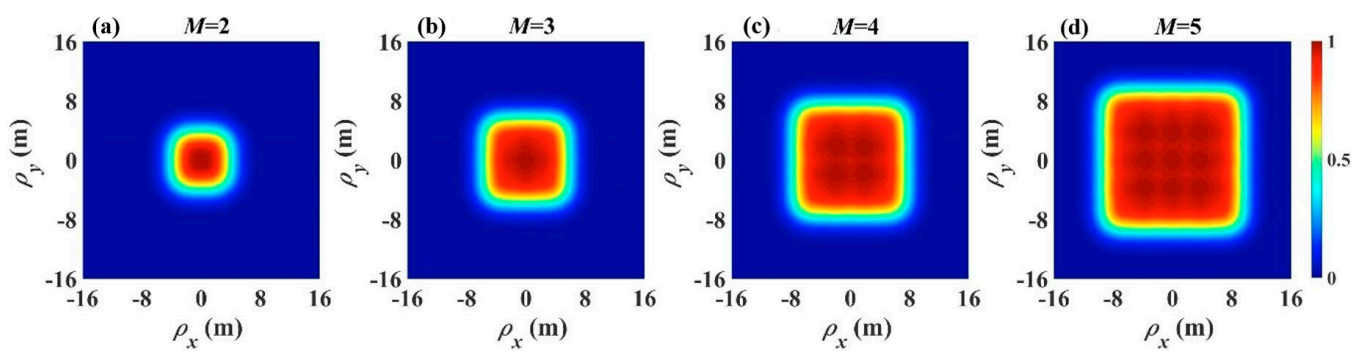

Figure 3. Spectral density distribution of the optical coherence vortex lattices in turbulent atmosphere at $z=5 \mathrm{~km}$ for different $M$ with $\lambda=532.8 \mathrm{~nm}, l=2, \delta_{0}=0.45 \mathrm{~mm}, \omega_{0}=1 \mathrm{~mm}, C_{n}^{2}=$ $10^{-13} \mathrm{~m}^{-2 / 3}$, and $d=0.3 \mathrm{~mm}$. (a) $M=2$; (b) $M=3$; (c) $M=4$; (d) $M=5$.
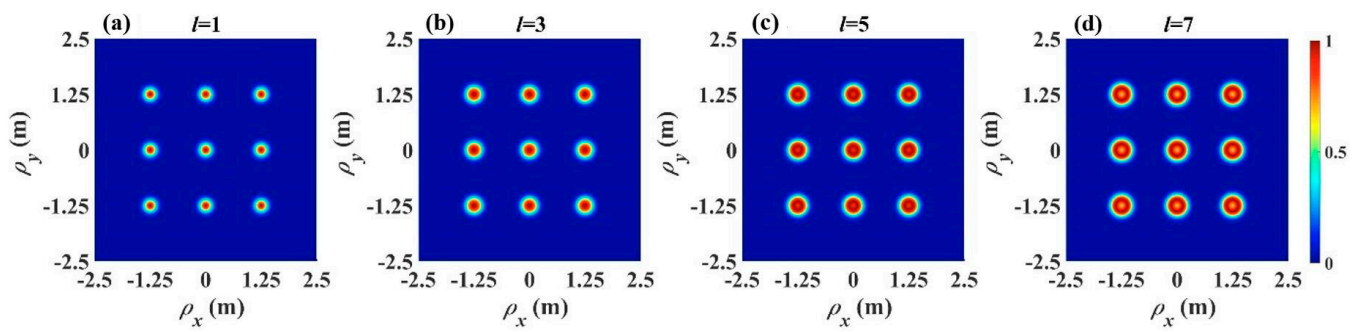

Figure 4. Spectral density distribution of the optical coherence vortex lattices in turbulent atmosphere at $z=500 \mathrm{~m}$ for different $l$ with $\lambda=532.8 \mathrm{~nm}, M=3, \delta_{0}=0.7 \mathrm{~mm}, \omega_{0}=1 \mathrm{~mm}, C_{n}^{2}=$ $10^{-13} \mathrm{~m}^{-2 / 3}$, and $d=1 \mathrm{~mm}$. (a) $M=2$; (b) $M=3$; (c) $M=4$; (d) $M=5$.

\section{2. $M^{2}$-Factor}

In this subsection, we explore the evolution of the normalized $M^{2}$-factor of the OCVLs in turbulent atmosphere.

Figure 5 displays the normalized $M^{2}$-factor of the OCVLs in turbulent atmosphere for several different topological charge $l$ and beam array order $M$ with $\lambda=532.8 \mathrm{~nm}, C_{n}^{2}=10^{-15} \mathrm{~m}^{-2 / 3}, d=$ $1 \mathrm{~mm}, \delta_{0}=5 \mathrm{~mm}$, and $\omega_{0}=1 \mathrm{~cm}$. Figure 5 a shows that as the normalized $M^{2}$-factor of the OCVLs remains invariant on propagation in free space $\left(C_{n}^{2}=0\right)$, this property represents the best beam 
quality. However, for the turbulent atmosphere, the normalized $M^{2}$-factor of the OCVLs increases on propagation, and its value is smaller than that of the Gaussian Schell-model (GSM) array $(l=0)$ when the propagation distance is fixed. Furthermore, the normalized $M^{2}$-factor increases with the decrease of the topological charge $l$. From Figure $5 \mathrm{~b}$, one can see that the normalized $M^{2}$-factor of the $\operatorname{OCVLs}(M>1)$ is smaller than that of the GSM vortex beam $(M=1)$, and the normalized $M^{2}$-factor increases as $M$ decreases. Figure 5 indicates that the OCVLs will be less affected by the negative effects of the turbulence for larger topological charge and larger beam array order from the view point of the normalized $M^{2}$-factor.
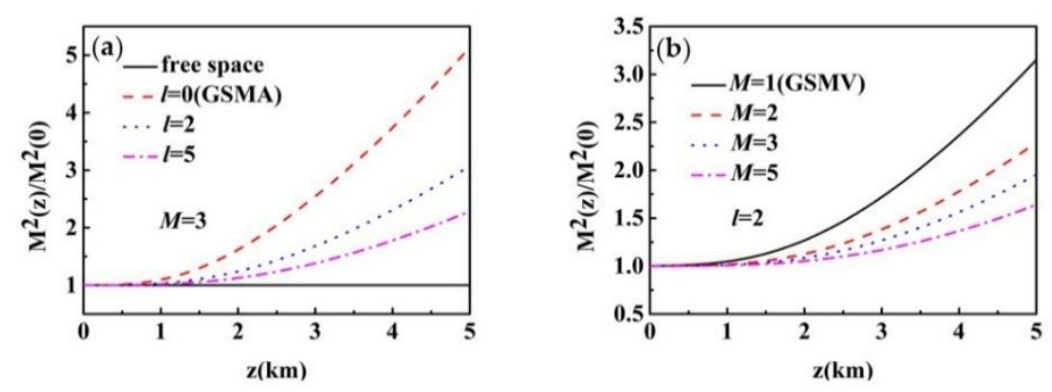

Figure 5. Normalized $M^{2}$-factor of the optical coherence vortex lattices in turbulent atmosphere for several different topological charge $l$ and beam array order $M$ with $\lambda=532.8 \mathrm{~nm}, C_{n}^{2}=10^{-15} \mathrm{~m}^{-2 / 3}, d=$ $1 \mathrm{~mm}, \delta_{0}=5 \mathrm{~mm}$, and $\omega_{0}=1 \mathrm{~cm}$. (a) $l=0,2,5(M=3) ;(\mathbf{b}) M=1,2,3,5(l=2)$.

\subsection{Beam Wander}

In this subsection, we explore the beam wander of the OCVLs in turbulent atmosphere for several different initial beam parameters.

Figure 6 shows the beam wander of the OCVLs in turbulent atmosphere for various $\delta_{0}$, relative distance $d$, topological charges $l$, and beam array order $M$ with $\lambda=532.8 \mathrm{~nm}, C_{n}^{2}=$ $10^{-15} \mathrm{~m}^{-2 / 3}$, and $\omega_{0}=1 \mathrm{~cm}$. From Figure 6 , it is found that the beam wander of the OCVLs in turbulent atmosphere increases as the coherence width $\delta_{0}$ increases, or as the relative distance $d$, topological charge $l$, and beam array order $M$ decrease, which means that the OCVLs are less affected by turbulence for large topological charge, large beam array order, large relative distance, and small coherence length from the view point of the beam wander.
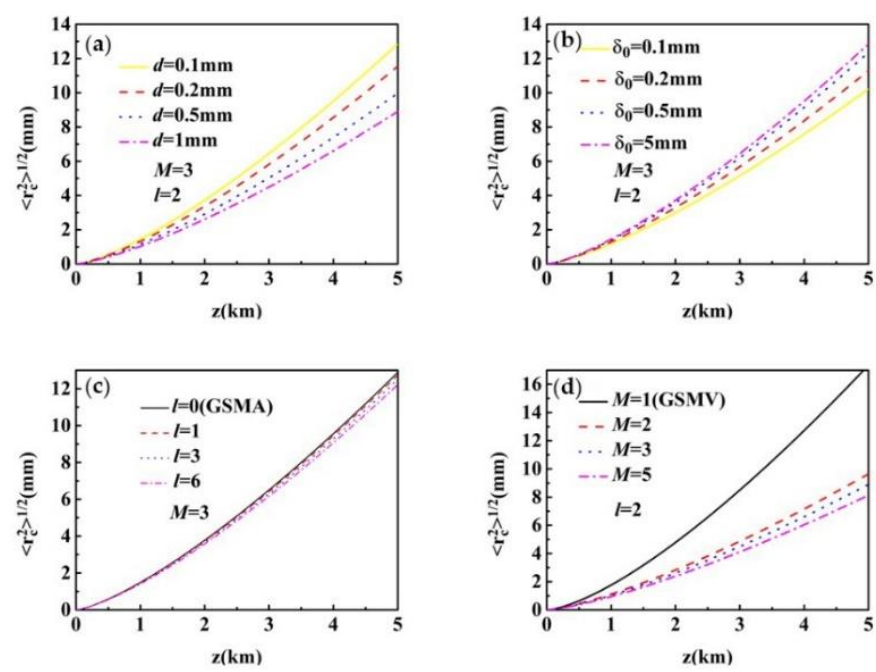

Figure 6. Beam wander of the optical coherence vortex lattices in turbulent atmosphere for various different values of $\delta_{0}$, relative distance $d$, topological charge $l$, and beam array order $M$ with $\lambda=$ $532.8 \mathrm{~nm}, C_{n}^{2}=10^{-15} \mathrm{~m}^{-2 / 3}$, and $\omega_{0}=1 \mathrm{~cm}$. (a) $d=0.1 \mathrm{~mm}, 0.2 \mathrm{~mm}, 0.5 \mathrm{~mm}, 1 \mathrm{~mm}(M=3, l=2)$; (b) $\delta_{0}=0.1 \mathrm{~mm}, 0.2 \mathrm{~mm}, 0.5 \mathrm{~mm}, 5 \mathrm{~mm}(M=3, l=2)$; (c) $l=0,1,3,6(M=3)$; (d) $M=1,2,3,5(l=2)$. 


\section{Conclusions}

We have formulated the propagation of the OCVLs in turbulent atmosphere by using the convolution approach, and we have explored propagation dynamics, such as the spectral density, the normalized $M^{2}$-factor, and the beam wander of the OCVLs in turbulent atmosphere. We have found that the beam distribution of the OCVLs changes from a single doughnut beam distribution to hollow array distribution on propagation, and finally becomes the Gaussian distribution in the far field. We also have found that the OCVLs with large topological charge, large beam array order, large relative distance, and small coherence length can mitigate the influence of atmospheric turbulence. Our findings are useful for free-space optical communications and information transfer.

Author Contributions: Data curation, Y.H.; writing (original draft), Y.H.; methodology, Y.H.; writing (review and editing), Y.Y., F.W., X.L., J.Z., J.Y., and Y.C.; formal analysis, X.L., J.Z., and J.Y.; supervision, Y.Y., F.W., L.L., and Y.C.; project administration, Y.C.

Funding: This research was funded by the National Natural Science Fund for Distinguished Young Scholar [grant number 11525418], the National Natural Science Foundation of China [grant numbers 91750201 and 11774251 and 11874046 and 11804198], the Priority Academic Program Development of Jiangsu Higher Education Institutions, and the Qing Lan Project of Jiangsu Province.

Conflicts of Interest: The authors declare no conflict of interest.

\section{References}

1. Ma, L.; Ponomarenko, S.A. Optical coherence gratings and lattices. Opt. Lett. 2014, 39, 6656-6659. [CrossRef] [PubMed]

2. Ponomarenko, S.A. Complex Gaussian representation of statistical pulses. Opt. Express 2011, 19, 17086-17091. [CrossRef] [PubMed]

3. Ma, L.; Ponomarenko, S.A. Free-space propagation of optical coherence lattices and periodicity reciprocity. Opt. Express 2015, 23, 1848-1856. [CrossRef] [PubMed]

4. Chen, Y.; Ponomarenko, S.A.; Cai, Y. Experimental generation of optical coherence lattices. Appl. Phys. Lett. 2016, 109, 061107. [CrossRef]

5. Liang, C.; Mi, C.; Wang, F.; Zhao, C.; Cai, Y.; Ponomarenko, S.A. Vector optical coherence lattices generating controllable far-field beam profiles. Opt. Express 2017, 25, 9872-9885. [CrossRef] [PubMed]

6. Mei, Z.; Zhao, D.; Korotkova, O.; Mao, Y. Gaussian schell-model arrays. Opt. Lett. 2015, 40, 5662-5665. [CrossRef] [PubMed]

7. Mei, Z.; Korotkova, O. Alternating series of cross-spectral densities. Opt. Lett. 2015, 40, 2473-2476. [CrossRef] [PubMed]

8. Wan, L.; Zhao, D. Optical coherence grids and their propagation characteristics. Opt. Express 2018, 26, 2168-2180. [CrossRef]

9. Jaksch, D.; Bruder, C.; Cirac, J.I.; Gardiner, C.W.; Zoller, P. Cold bosonic atoms in optical lattices. Phys. Rev. Lett. 1998, 81, 3108-3111. [CrossRef]

10. Ostrovskaya, E.; Kivshar, Y. Photonic crystals for matter waves: Bose-Einstein condensates in optical lattices. Opt. Express 2004, 12, 19-29. [CrossRef]

11. Betzig, E. Excitation strategies for optical lattice microscopy. Opt. Express 2005, 13, 3021-3036. [CrossRef] [PubMed]

12. Bloch, I. Ultracoldquantum gases in optical lattices. Nat. Phys. 2005, 1, 23-30. [CrossRef]

13. Lu, W.; Liu, L.; Sun, J. Change in degree of coherence of partially coherent electromagnetic beams propagating through atmospheric turbulence. Opt. Commun. 2007, 271, 1-8. [CrossRef]

14. Wang, F.; Cai, Y.; Eyyuboglu, H.T.; Baykal, Y. Partially coherent elegant Hermite-Gaussian beam in turbulent atmosphere. Appl. Phys. B 2011, 103, 461-469. [CrossRef]

15. Gbur, G.; Wolf, E. Spreading of partially coherent beams in random media. J. Opt. Soc. Am. A 2002, 19, 1592-1598. [CrossRef]

16. Yuan, Y.; Cai, Y.; Qu, J.; Eyyuboglu, H.T.; Baykal, Y.; Korotkova, O. M $^{2}$-factor of coherent and partially coherent dark hollow beams propagating in turbulent atmosphere. Opt. Express 2009, 17, 17344-17356. [CrossRef] [PubMed] 
17. Wang, F.; Cai, Y. Second-order statistics of a twisted Gaussian Schell-model beam in turbulent atmosphere. Opt. Express 2010, 18, 24661-24672. [CrossRef]

18. Dan, Y.; Zhang, B. Beam propagation factor of partially coherent flat-topped beams in a turbulent atmosphere. Opt. Express 2008, 16, 15563-15575. [CrossRef]

19. Gbur, G.; Wolf, E. The rayleigh range of Gaussian Schell-model beams. J. Mod. Opt. 2001, 48, $1735-1741$. [CrossRef]

20. Wang, F.; Cai, Y.; Eyyuboglu, H.T.; Baykal, Y. Twist phase-induced reduction in scintillation of a partially coherent beam in turbulent atmosphere. Opt. Lett. 2012, 37, 184-186. [CrossRef]

21. Baykal, Y.; Eyyuboglu, H.T.; Cai, Y. Scintillations of partially coherent multiple Gaussian beams in turbulence. Appl. Opt. 2009, 48, 1943-1954. [CrossRef] [PubMed]

22. Yuan, Y.; Liu, L.; Wang, F.; Chen, Y.; Cai, Y.; Qu, J.; Eyyuboğlu, H.T. Scintillation index of a multi-Gaussian Schell-model beam. Opt. Commun. 2013, 305, 57-65. [CrossRef]

23. Liu, X.; Yu, J.; Cai, Y.; Ponomarenko, S.A. Propagation of optical coherence lattices in the turbulent atmosphere. Opt. Lett. 2016, 41, 4182-4185. [CrossRef] [PubMed]

24. Chen, Y.; Wang, F.; Zhao, C. Experimental demonstration of a Laguerre Gaussian correlated Schell-model vortex beam. Opt. Express 2014, 22, 5826-5838. [CrossRef] [PubMed]

25. Ng, J.; Lin, Z.; Chan, C.T. Theory of optical trapping by an optical vortex beam. Phys. Rev. Lett. 2010, 104, 103601. [CrossRef] [PubMed]

26. Grier, D. A revolution in optical manipulation. Nature 2003, 424, 810-816. [CrossRef] [PubMed]

27. Li, X.; Ma, H.; Zhang, H.; Tai, Y.; Li, H.; Tang, M.; Wang, J.; Tang, J.; Cai, Y. Close-packed optical vortex lattices with controllable structures. Opt. Express 2018, 26, 22965-22975. [CrossRef] [PubMed]

28. Yuan, Y.; Liu, D.; Zhou, Z.; Xu, H.; Qu, J.; Cai, Y. Optimization of the probability of orbital angular momentum for Laguerre-Gaussian beam in Kolmogorov and Non-Kolmogorov turbulence. Opt. Express 2018, 26, 21861-21871. [CrossRef]

29. Gbur, G.; Tyson, R. Vortex beam propagation through atmospheric turbulence and topological charge conservation. J. Opt. Soc. Am. A 2008, 25, 225-230. [CrossRef]

30. Gori, F.; Santarsiero, M.; Borghi, R.; Vicalvi, S. Partially coherent sources with helicoidal modes. J. Mod. Opt. 1998, 45, 539-554. [CrossRef]

31. Liu, X.; Shen, Y.; Liu, L.; Wang, F.; Cai, Y. Experimental demonstration of vortex phase-induced reduction in scintillation of a partially coherent beam. Opt. Lett. 2013, 38, 5323-5326. [CrossRef] [PubMed]

32. Bogatyryova, G.V.; Fel'de, C.V.; Polyanskii, P.V.; Ponomarenko, S.A.; Soskin, M.S.; Wolf, E. Partially coherent vortex beams with a separable phase. Opt. Lett. 2003, 28, 878-880. [CrossRef] [PubMed]

33. Ponomarenko, S.A. A class of partially coherent beams carrying optical vortices. J. Opt. Soc. Am. A 2001, 18, 150-156. [CrossRef]

34. Palacios, D.; Maleev, I.; Marathay, A.; Swartzlander, G.A. Spatial correlation singularity of a vortex field. Phys. Rev. Lett. 2004, 92, 143905. [CrossRef] [PubMed]

35. Wang, F.; Cai, Y.; Korotkova, O. Partially coherent standard and elegant Laguerre-Gaussian beams of all orders. Opt. Express 2009, 17, 22366-22379. [CrossRef] [PubMed]

36. Wang, F.; Zhu, S.; Cai, Y. Experimental study of the focusing properties of a Gaussian Schell-model vortex beam. Opt. Lett. 2011, 36, 3281-3283. [CrossRef] [PubMed]

37. Zhao, C.; Dong, Y.; Wang, Y.; Wang, F.; Zhang, Y.; Cai, Y. Experimental generation of a partially coherent Laguerre-Gaussian beam. Appl. Phys. B 2012, 109, 345-349. [CrossRef]

38. Zhao, C.; Wang, F.; Dong, Y.; Han, Y.; Cai, Y. Effect of spatial coherence on determining the topological charge of a vortex beam. Appl. Phys. Lett. 2012, 101, 261104. [CrossRef]

39. Perez-Garcia, B.; Yepiz, A.; Hernandez-Aranda, R.I.; Forbes, A.; Swartzlander, G.A. Digital generation of partially coherent vortex beams. Opt. Lett. 2016, 41, 3471-3474. [CrossRef]

40. Guo, L.; Chen, Y.; Liu, X.; Liu, L.; Cai, Y. Vortex phase-induced changes of the statistical properties of a partially coherent radially polarized beam. Opt. Express 2016, 24, 13714-13728. [CrossRef]

41. Liu, X.; Liu, L.; Chen, Y.; Cai, Y. Partially coherent vortex beam: From theory to experiment. Vortex Dynamics and Optical Vortices; Pérez-de-Tejada, H., Ed.; InTech-open science: London, UK, 2017.

42. Liu, X.; Peng, X.; Liu, L.; Wu, G.; Zhao, C.; Wang, F.; Cai, Y. Self-reconstruction of the degree of coherence of a partially coherent vortex beam obstructed by an opaque obstacle. Appl. Phys. Lett. 2017, 110, 181104. [CrossRef] 
43. Zeng, J.; Liu, X.; Wang, F.; Zhao, C.; Cai, Y. Partially coherent fractional vortex beam. Opt. Express 2018, 26, 26830-26844. [CrossRef] [PubMed]

44. Liu, X.; Liu, L.; Peng, X.; Liu, L.; Wang, F.; Cai, Y. Partially coherent vortex beam with periodical coherence properties. J. Quant. Spectrosc. Radiat. Transfer 2019, 222-223, 138-144. [CrossRef]

45. Gori, F.; Santarsiero, M. Devising genuine spatial correlation functions. Opt. Lett. 2007, 32, 3531-3533. [CrossRef] [PubMed]

46. Wang, F.; Korotkova, O. Convolution approach for beam propagation in random media. Opt. Lett. 2016, 41, 1546-1549. [CrossRef] [PubMed]

47. Wang, F.; Li, J.; Martinez-Piedra, G.; Korotkova, O. Propagation dynamics of partially coherent crescent-like optical beams in free space and turbulent atmosphere. Opt. Express 2017, 25, 26055-26066. [CrossRef] [PubMed]

48. Zhu, S.; Cai, Y.; Korotkova, O. Propagation factor of a stochastic electromagnetic Gaussian Schell-model beam. Opt. Express 2010, 12, 12587-12598. [CrossRef] [PubMed]

49. Liu, X.; Wang, F.; Wei, C.; Cai, Y. Experimental study of turbulence-induced beam wander and deformation of a partially coherent beam. Opt. Lett. 2014, 39, 3336-3339. [CrossRef]

50. Yu, J.; Chen, Y.; Liu, L.; Liu, X.; Cai, Y. Splitting and combining properties of an elegant Hermite-Gaussian correlated Schell model beam in Kolmogorov and non-Kolmogorov turbulence. Opt. Express 2015, 23, 13467-13481. [CrossRef]

51. Dan, Y.; Zhang, B. Second moments of partially coherent beams in atmospheric turbulence. Opt. Lett. 2009, 34, 563-565. [CrossRef]

52. Martínez-Herrero, R.; Piquero, G.; Mejías, P.M. Beam quality changes of radially and azimuthally polarized fields propagating through quartic phase plates. Opt. Commun. 2008, 281, 756-759. [CrossRef]

53. Santarsiero, M.; Gori, F.; Borghi, R.; Cincotti, G.; Vahimaa, P. Spreading properties of beams radiated by partially coherent Schell-model sources. J. Opt. Soc. Am. A 1999, 1, 106-112. [CrossRef]

54. Huang, Y.; Zeng, A.; Gao, Z.; Zhang, B. Beam wander of partially coherent array beams through non-Kolmogorov turbulence. Opt. Lett. 2015, 40, 1619-1622. [CrossRef] [PubMed]

(C) 2018 by the authors. Licensee MDPI, Basel, Switzerland. This article is an open access article distributed under the terms and conditions of the Creative Commons Attribution (CC BY) license (http:/ / creativecommons.org/licenses/by/4.0/). 